\title{
THE EPIDEMIOLOGY OF MULTIPLE SCLEROSIS IN ROMANIA AND ACROSS CENTRAL AND SOUTHEASTERN EUROPE - A HISTORICAL REVIEW
}

\author{
A. Cornea, C. Rosca, A. Petre, M. Simu \\ II ${ }^{\text {nd }}$ Departament of Neurology, "V. Babes” University of Medicine and Pharmacy, Timisoara
}

\begin{abstract}
Background. Multiple sclerosis (MS) has a variable clinical and pathological course and pace, which eventually turns over years of that progression into a major source of disability especially among young adults.

The regional incidence and prevalence data course are of utmost interest (i.e they tend to be higher in Europe within the last decade) mirroring diagnosis improvement, earlier and better treatment choices and an improved physio-pathological insight as shown by most recent studies. (1)

The incidence and prevalence data tend to be higher in Europe within the last decade and are explained by diagnosis improvement and earlier and better treatment solutions for the patients mirrored by most recent studies. (1) The objective of the present study is to assess data available concerning the prevalence of multiple sclerosis in Romania and in Central and Southeastern Europe in a regional view.

Material and method. A literature search has been performed in PubMed, DIMDI, Web of Science, and Google Scholar to retrieve population based studies in Central and Eastern Europe and epidemiological reviews through years 1960-2015. We searched the term "MS" combined with one of the following: "epidemiology" or "incidence" or "prevalence". We also did a manual search of reference list of the relevant articles.

Results. 38 studies met the inclusion criteria and were analyzed. The epidemiologic data available for Central and Eastern Europe during the period 1960-2015 support the heterogeneity both of this region's resident population and of the studies design, making any global data assessment difficult, with a questionable reliability, therefore justifying a newer reappraisal.
\end{abstract}

Keywords: multiple sclerosis, incidence, prevalence, pathological course

\section{INTRODUCTION}

Multiple sclerosis (MS) is a chronic immune mediated demyelinating disease with a neurodegenerative component and unknown etiology. Development of this disease is associated with multiple factors which include genetic factors (involving predominantly the Caucasians, focusing over certain communities), environmental factors (the North South gradient, vitamin D deficiency), infectious agents (as Epstein-Barr and infectious mononucleosis) and autoimmune conditions association $(2,3)$ Multiple sclerosis (MS) represents the most common cause of disability in young adults through the world and almost half of them are in Europe (1).

Epidemiologic studies conducted in several European countries revealed regions with prevalence as high as $252 / 10^{5}$ in region of Binzer, Sweden
(1994) to values of $15 / 10^{5}$ in Garcia, Spain (1989), reasoning in favor of latitude gradient theory (1). Migration and heterogeneity of European population determines steep transitions from lower to very high epidemiologic indices over limited areas as in Sicily (4) compared with Palermo, Italy $(1,5)$.

The prevalence data also reveals West to East gradient with higher prevalence and incidence rate in UK 203/100,000 respectively 9,64/10 (6) per year and lower values toward East in countries as Albania and Romania where prevalence is around 19,6-25/100,000 (7,8). We have focused in a geographical space, which frames Romanian borders and analyzed the data concerning prevalence changing estimates since 1960 to 2015 for this area versus similar available data from the neighboring countries. 


\section{MATERIAL AND METHOD}

\section{Screening and eligibility criteria}

We have selected the studies conducted in the South Central European countries: Poland, the Czech Republic, Hungary, Romania, Moldova, Bulgaria Slovakia, Croatia, Serbia, Bosnia Herzegovina, Montenegro, Macedonia, Albania, the Ukraine and Belarus, the western part of the Russian federation.

We have removed the duplicate records and assessed the abstracts with respect to primary focus on prevalence and incidence of MS in selected countries. We have reviewed the full articles representative for our analysis and centralised the results. We used the PRISMA flow Diagram for reviewed literature (9) in Table 1.

\section{Data extraction and statistical analysis}

We have recorded the following data: the study period, location, diagnostic criteria, number of patients, female/male ratio, average age of the study population incidence and prevalence estimates, if data were available.

\section{RESULTS}

We retrieved from PubMed, DIMDI and Google Scholar 1,928 respectively, 306 studies and epidemiological reviews (cohorts, case-control, crosssectional studies and case-series in which prevalence, incidence of MS data were provided). 38 studies were selected after considering the title and abstract as satisfying the selection criteria. They were classified by the period and the diagnostic criteria used to ascertain the cases in three periods 1960-1985 (Allison and Millar or Bauer criteria); 1986-2004 (Poser criteria) and 2005-2015 (MacDonald criteria.)

The most constant epidemiologic parameter that appears through the papers was prevalence, which summarized several factors that impacted the accuracy of information. For the first fifteen years of

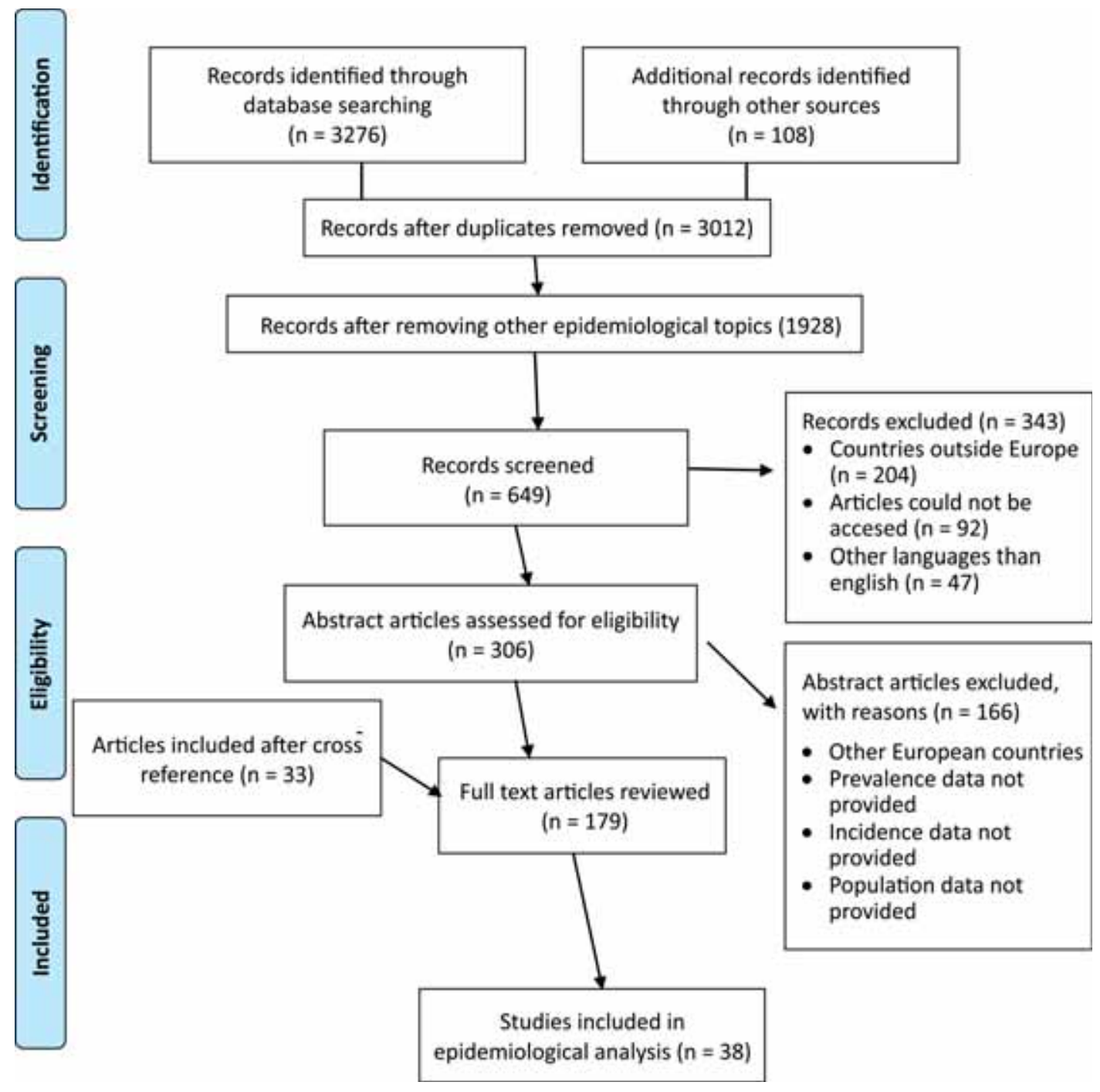




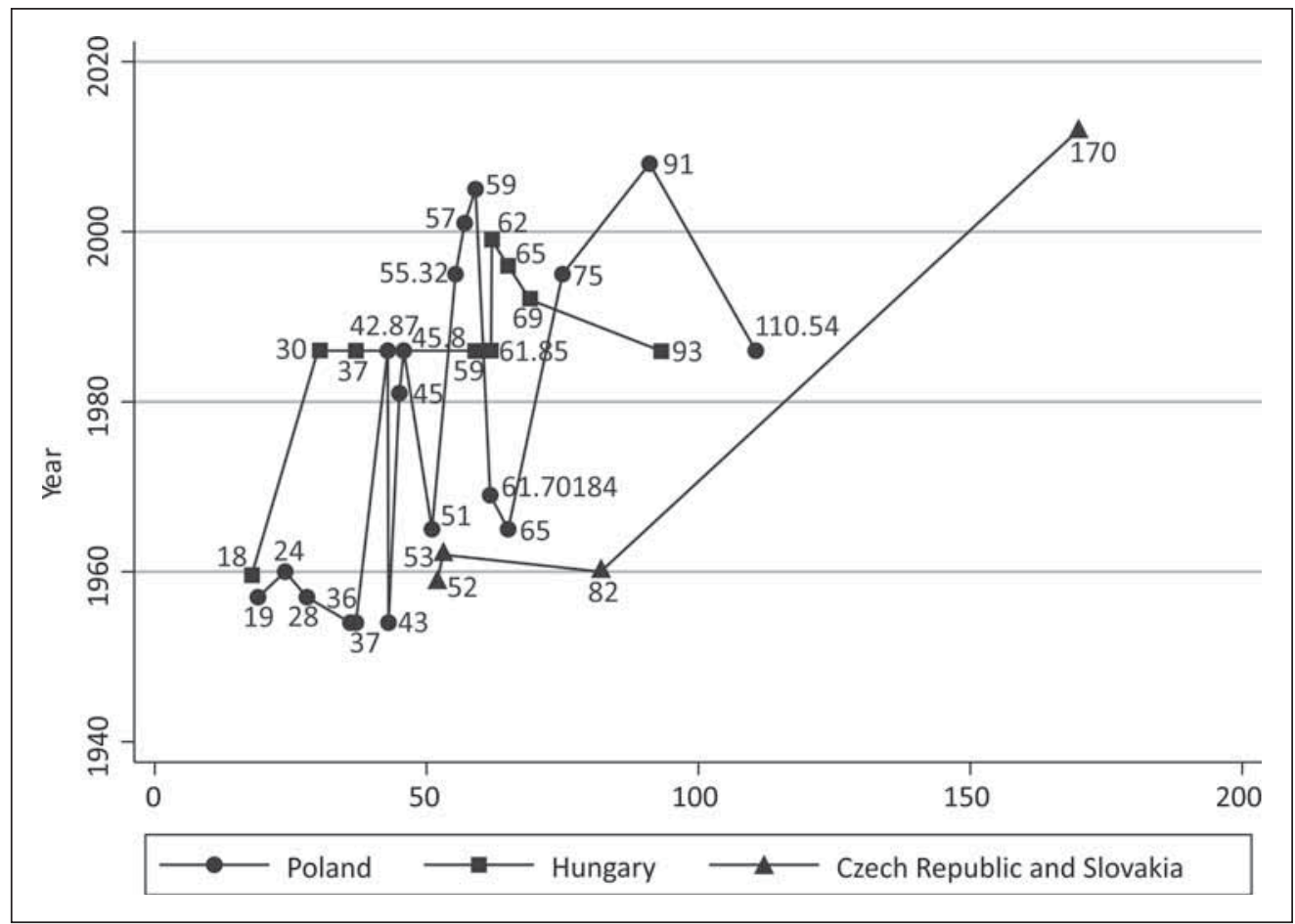

GRAPHIC 1. Evolution of crude prevalence data in Poland, Czech Republic, Slovakia, and Hungary

MS epidemiologic studies, the major discrepancies resulted from inaccurate diagnosis, ascertainment difficulties of the cases and short survival time (10).

\section{Poland, Czech and Slovakia, Hungary (Graphic 1)}

The prevalence of MS in Poland during 19651981 has been evaluated mostly in the north central region, were based mainly on hospital records, and included Poznan, Bydgoszcz and Krosno and ranged from 24 to 65 with a tendency to decrease from 52 to 45 in Poznan area from 1965 till 1981, probably due to a better ascertainment of cases (11). The lowest values of $24 / 100,000$ were retrieved in Pruskow in 1960 (12).

The prevalence documented in the following studies sustained the increasing number of diagnosed patients as well as the longer duration of the disease. In 1995 in the province of Szczecin Northwest Poland, prevalence was calculated at $110,54 / 100,000$ (13); the studies of 2005 data retrieved varied from $45-95 / 100,000$ and the last survey inclusion criteria was definite MS according to McDonald criteria and restricted the evaluated patients to those living in Szczecin area $(14,15)$.

We have found five studies regarding Hungarian prevalence and incidence of multiple sclerosis. The earliest data indicate a prevalence of $18 / 100,000$ (16-21) in Budapest and surrounding areas, in 1960, based on hospital records (16). The frequen- cy of MS cases increased as indicated by prevalence results from the following studies, in Baranya County, 1983 - 37/100,000 and in Fejer County, 1992 69/100,000 mainly, based on hospital diagnostic data (17). The epidemiologic study published in 1997 in the region of Szeged, South Hungary, pointed to a medium to high prevalence indices of $65 / 100,000$ on the prevalence day $1 / 12 / 1996$. The incidence of MS new cases through 1993-1996 was $7 / 100,000$. It was in line with prevalence frequencies from the higher risk group countries of North Europe (17). The subsequent epidemiological survey conducted in the Szeged region, 1999 evidenced a prevalence of $62 / 100,000$ and an incidence of $5.5 / 100,000$, confirming the previously obtained data (18).

For Czech Republic and Slovakia, the early epidemiologic data are represented by four studies conducted through 1959-1978 and indicating prevalence which range from 53-82/100,000, the highest values being found in Prague (19). In 1984, the prevalence of MS was 71/100,000 and followed an increasing tendency towards $89 / 100,000$ in three districts of Bohemian area (20).

\section{Croatia, Slovenia, Bosnia and Herzegovina,} Yugoslavia, Bulgaria, Albania, and Greece (Graphic 2)

Croatia is a country with consistent prevalence and incidence of MS data gathered by several stud- 


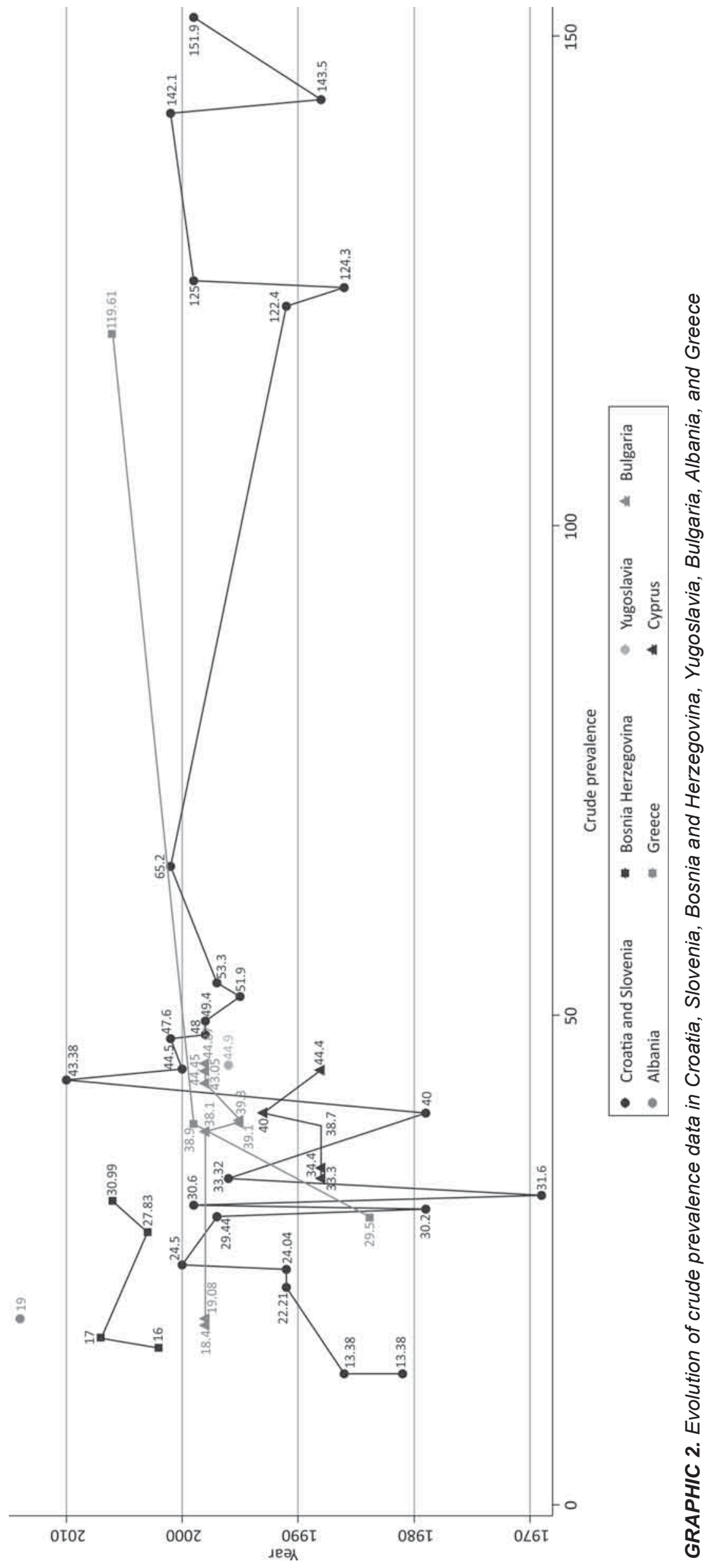


ies conducted through 1969-1980 in the following areas: Rijeka, Gorski Kotar, Bol, Split, Zagreb, Istria - and revealed prevalence numbers ranging from 5 to 84/100,000, with the lowest value in Bol, Split and the Highest in Rijeka. (21)

The higher prevalence estimates (144-152/ 100,000) evidenced in Gorski Kotar region, demonstrates an increase trend in data collected by Sepcic in 2001, Peterlin in 2006 and Perkovic in 2010 $(22,23)$ in both Croatian and Yugoslavian zone.

The data about Bosnia Herzegovina has been mainly ascertained in 2003 and 2006 by two descriptive epidemiological studies, which situate the country in the area of moderate risk of multiple sclerosis. The prevalence values obtained are 25.15/ 100,000 in 2003 and 30.99/100,000 in 2006 and support the increasing number of multiple sclerosis cases $(24,25)$. MacDonald's criteria were used for both studies for the selection of the patients.

Bulgaria is considered a country with low/moderate risk of multiple sclerosis as surveys run by Milanov in 1994 and then 1998 evidenced a crude prevalence of $39.1-19.08-38 / 100,000$ in Trojan, Sofia and Samokov areas $(1,26,27)$. Both studies have verified the ascertainment of cases from multiple sources - hospital and outpatient clinics and defined the MS diagnostic criteria which were used by Poser for both evaluations 1994 respectively, 1998. The number of MS patient was calculated by sex and by age groups. The ratio women: men was provided attaining values of $1.4: 1$ respectively
1.9:1 in Sofia region $(26,27)$. Another strength point of the study was evaluation of ethnic rural vs. urban Gypsies population evidencing the ethnic differences between Roma and non Roma population in terms of prevalence: 10.8 in Samokov region (rural) and 19.08 in Sofia (urban area) (1).

Evolution of prevalence of multiple sclerosis cases in North and West region of Greece has been assessed by Milonas in 1984 and Piperidou in 1999, recording a prevalence rate of 29.5 respectively $38.9 / 100,000(28,29)$. This classifies Greece in the medium frequency zone.

MS prevalence in Albania is represented by the study of multiple sclerosis patients from Kosovo in the period between 2003-2012, indicating a rate of $19.6 / 100,000$ prevalence and an incidence rate of $0.95 / 1,000,000 /$ year (30).

\section{Western Russia, Ukraine, Estonia, Moldavia} (Graphic 3)

Data collected from Russia during different time frames and different zones, evidence a moderate risk zone for MS, the highest prevalence of 20,4 and 29/100,000 being documented in 1995 in "Pricarpathie region" (a region in western Ukraine ) and respectively, in 1987 in South Amur region (31).

In Estonia the prevalence data for native Estonians (55/100,000 inhabitants), 29/100,000 inhabitants for the Russian population, and 42/100,000 for

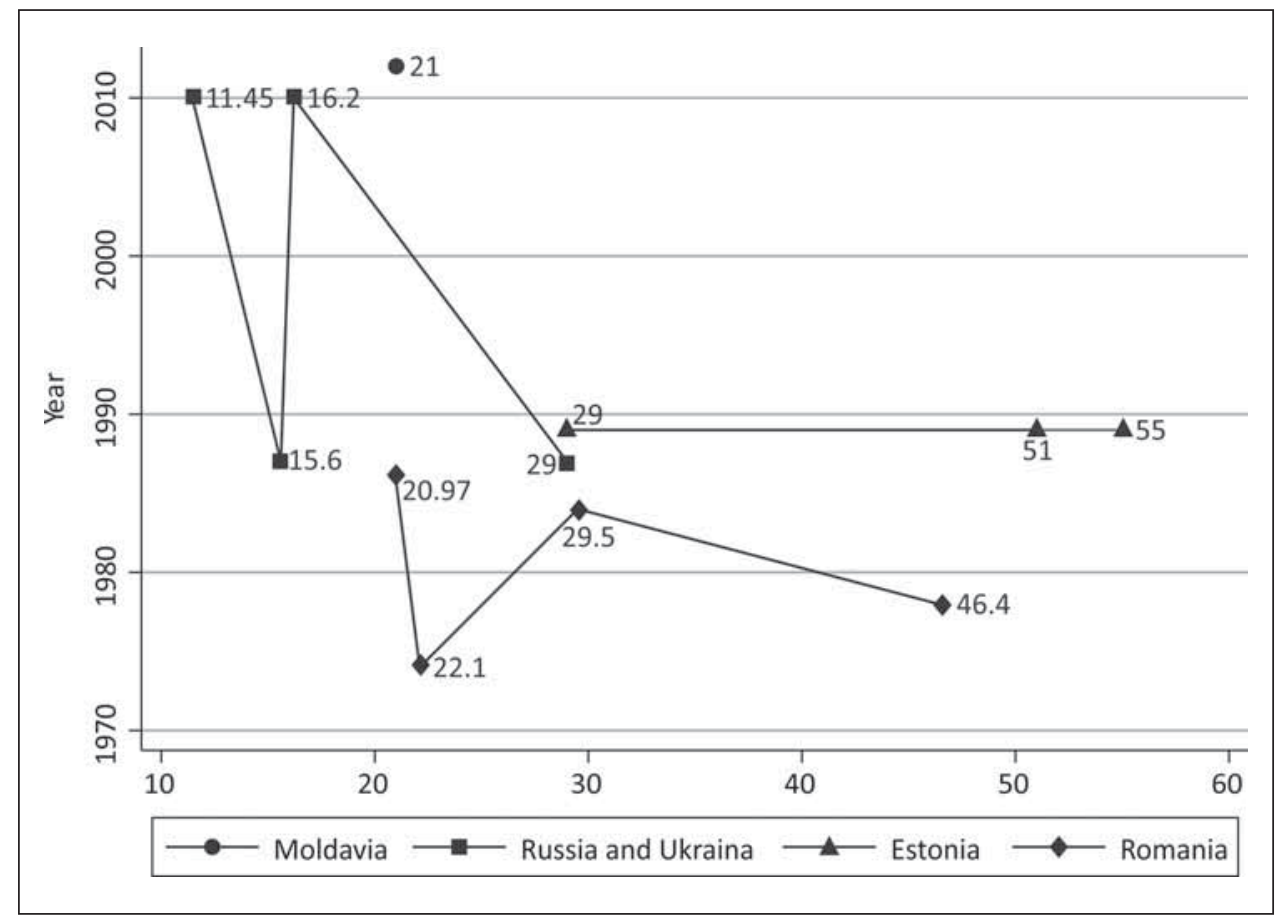

GRAPHIC 3. Evolution of crude prevalence data in Western Russia, Ukraine, Estonia, Moldavia, Romania 
the other nationalities qualified this country within the "Fennoscandian zone" (the South-Western of Finland, the North-Eastern Sweden and Western Norway), as a region of high prevalence rates ranging between 87 to $104 / 100,000$ inhabitants for multiple sclerosis (32-35)

A MS Moldavian population survey has been led in 2012 by Marcoci et al., and evidenced a crude prevalence of 21/100,000 with a women: man ratio of 1.7: 1 (36). The study fulfills the quality criteria concerning diagnostic inclusion criteria, MS population characterization, age adjusted prevalence and incidence estimates, women to men ratio.

\section{Romania}

The studies led by Verdes in Bucharest in 1978 and Morariu in Transylvania in 1974 evidenced prevalence rates of 46.4 in Bucharest area and 22.1 for Transylvania respectively. $(37,38)$ These data are not concordant with the expected geographical North latitude distribution of MS cases across Europe, nevertheless the Transylvanian estimates remain similar within the following epidemiologic evaluations done by Becus and Balasa for Mures County. (8)

Data obtained point out to a prevalence of 26.1/100,000 for Mures county in 2006, evidencing a slight increase of the cases frequency. (39)

\section{DISCUSSIONS}

This review has focused on prevalence data gathered from studies and epidemiological survey that inquired frequency of MS across Central and South Eastern Europe between 1960 and 2015. We consider 3 successive time frames within the searched period due to the diagnostic criteria used to document the disease.

All major epidemiologic surveys carried in the first period 1960-1985 had the disadvantage of using a broader spectrum of diagnosis criteria (Allison and Millar or Bauer criteria), which overestimated the prevalence data. The European distribution of MS is described as containing areas of prevalence: low $<5 / 100,000$, medium - ranging from 5-29/100,000, and high - above 30/100,000 (40). This highest prevalence zone was found between 45-65 grades north latitude for Europe, followed by the medium prevalence zone around 40 grades North latitude, while the area around 35 grades North latitude was considered a low prevalence zone i.e.- south Europe and Mediterranean zone.
The studies that document MS cases through 1985 to 2000 used Poser criteria for case diagnosis and inclusion and many of the data obtained from this period displayed a slight decrease of the epidemiologic parameters. The studies are highly heterogeneous, and the need of a unifying concept in design of the study develops. Epidemiologic data represent the key information to untangle the etiology and pathology of MS. The geographic theory was unsustainable by latitude and prevalence factors only, the influence of the genetic and environment component pointing towards associated risk factors (2).

Over this period of time several studies have been conducted in some of European countries, with refined and improved case documentation. Poland, Czech Republic and Croatia were better ranked among the countries we have concentrated on, but unlikely some of the Western and North Europe countries (Norway, Sweden, Denmark) they fail to offer MS prevalence and incidence data at a national level. Most of the studies involved small areas and/or communities that were followed up over repeated periods of time by different methods $(1,10,32)$.

The third period uses Mc Donalds criteria for case inclusion and defines the aim to communicate epidemiologic parameters in a standardized way for the incidence and prevalence to alleviate the comparison among studies (41). As a result the quality of the studies improved and the surveys evaluated, as a step further in the approach, also the genetic side of the disease's epidemiology. This aim is mainly achieved by initiating and completing national multiple sclerosis registries (42).

The Romanian published studies were performed according to each period's requirements for data assessment, in an attempt to be as thoroughly as possible using various MS parameters. The study conducted by Verdes in 1978 across Bucharest area and the one led by Morariu in 1974 in Transylvania were considered as B level of evidence by Kurtze in an epidemiological review conducted in 1975, nevertheless due to the paucity of data concerning the rest of the Romanian territory our country data remains to "considerable question" at the time. $(42,43)$.

The study conducted by Becus in 1994 in Mures County was included and ranked as 4/7 quality standards points in a 2013 Kingwell thorough review about European incidence and prevalence of multiple sclerosis (1).

Apart of this data the initiation of the national MS registry (a recent ongoing project of the Roma- 
nian Neurological Society) is an important initiative meant to standardize epidemiological data appraisal of MS in our country in order to make them reliable and useful.

\section{REFERENCES}

1. Kingwell E., Marriott J.J., Jetté N., Pringsheim T., Makhani N., Morrow S.A., et al. - Incidence and prevalence of multiple sclerosis in Europe: a systematic review. BMC Neurol. 2013; 13:128.

2. Hogancamp W.E., Rodriguez M., Weinshenker B.G. - The epidemiology of multiple sclerosis. Mayo Clin Proc. 1997; 72(9):871-8.

3. Milo R., Kahana E. - Multiple sclerosis: geoepidemiology, genetics and the environment. Autoimmun Rev. 2010; 9(5):A387-94.

4. Nicoletti A., Patti F., Lo Fermo S., Sorbello V., Reggio E., Maimone D., et al. - Possible increasing risk of multiple sclerosis in Catania, Sicily. Neurology. 2005; 65(8):1259-63.

5. Gross K., Kokk A., Kaasik A.E. - Prevalence of MS in south Estonia. Evidence of a new border of the Fennoscandian focus. Acta Neurol Scand. 1993; 88(4):241-6.

6. Mackenzie I.S., Morant S.V., Bloomfield G.A., MacDonald T.M., O'Riordan J. - Incidence and prevalence of multiple sclerosis in the UK 1990-2010: a descriptive study in the General Practice Research Database. J Neurol Neurosurg Psychiatry. 2014; 85(1):76-84.

7. Kruja J., Beghi E., Zerbi D., Dobi D., Kuqo A., Zekja I., et al. High prevalence of major neurological disorders in two Albanian communities: results of a door-to-door survey. Neuroepidemiology. 2012; 38(3):138-47.

8. Becuş T., Popoviciu L. - Epidemiologic survey of multiple sclerosis in Mureş County, Romania. Rom J Neurol Psychiatry. 1994; 32(2):115-22.

9. Liberati A., Altman D.G., Tetzlaff J., Mulrow C., Gøtzsche P.C, loannidis J.P., et al. - The PRISMA statement for reporting systematic reviews and meta-analyses of studies that evaluate health care interventions: explanation and elaboration. J Clin Epidemiol. 2009; 62(10):e1-34.

10. Koch-Henriksen N., Sørensen P.S. - The changing demographic pattern of multiple sclerosis epidemiology. Lancet Neurol. 2010; $9(5): 520-32$.

11. Wender M., Kowal P., Pruchnik-Grabowska D., Hertmanowska H., Zielińska M., Namysł I. - [Epidemiology of multiple sclerosis in the Wielkopolska region]. Przegl Epidemiol. 1986; 40(4):387-97.

12. Cendrowski W.S. - Multiple sclerosis in a small urban community in central Poland. J Neurol Sci. 1965; 2(1):82-6.

13. Potemkowski A. - [Epidemiology of multiple sclerosis in the region of Szczecin: prevalence and incidence 1993-1995]. Neurol Neurochir Pol. 1999; 33(3):575-85

14. Potemkowski A. - [An epidemiologic survey of multiple sclerosis in the Szczecin province in Poland]. Przegl Epidemiol. 2001; 55(3):33141.

15. Potemkowski A. - Epidemiological characteristics of multiple sclerosis in the district of Szczecinek, Northwestern Poland: 13 year observation, 1993-2005. In: Szpernalowska D, editor. 2010.

16. Kurtzke J.F. - A reassessment of the distribution of multiple sclerosis. Part one. Acta Neurol Scand. 1975; 51(2):110-36.

17. Bencsik K., Rajda C., Klivényi P., Járdánházy T., Vécsei L. - The prevalence of multiple sclerosis in the Hungarian city of Szeged. Acta Neurol Scand. 1998; 97(5):315-9.

18. Bencsik K., Rajda C., Füvesi J., Klivényi P., Járdánházy T., Török M., et al. - The prevalence of multiple sclerosis, distribution of clinical forms of the disease and functional status of patients in Csongrád County, Hungary. Eur Neurol. 2001; 46(4):206-9.

19. Randomised double-blind placebo-controlled study of interferon beta-1a in relapsing/remitting multiple sclerosis. PRISMS (Prevention of Relapses and Disability by Interferon beta-1a Subcutaneously in Multiple Sclerosis) Study Group. Lancet. 1998; 352(9139):1498-504.
Amalia Cornea acknowledges support from Project POSDRU/159/1.5/S/136893

20. Rosati G. - Descriptive epidemiology of multiple sclerosis in Europe in the 1980s: a critical overview. Ann Neurol. 1994; 36 Suppl 2:S164-74.

21. Bjørnevik K., Riise T., Casetta I., Drulovic J., Granieri E., Holmøy T., et al. - Sun exposure and multiple sclerosis risk in Norway and Italy: The EnvIMS study. Mult Scler. 2014; 20(8):1042-9.

22. Perković O, Jurjević A, Rudez J, Antoncić I, Bralić M, Kapović M. - The town of Cabar, Croatia, a high risk area for multiple sclerosis--analytic epidemiology of dietary factors. Coll Antropol. 2010; 34 Suppl 2:135-40.

23. Peterlin B., Ristić S., Sepcić J., Vracko B.K., Rako A., Lovrecić L., et al. - Region with persistent high frequency of multiple sclerosis in Croatia and Slovenia. J Neurol Sci. 2006; 247(2):169-72.

24. Klupka-Sarić I, Ristić S, Sepcić J, Kapović M, Peterlin B, Materljan E, et al. - Epidemiology of multiple sclerosis in western Herzegovina. Clin Neurol Neurosurg. 2007; 109(9):779-83.

25. Klupka-Sarić I., Galić M. - Epidemiology of multiple sclerosis in western Herzegovina and Herzegovina--Neretva Canton, Bosnia and Herzegovina. Coll Antropol. 2010; 34 Suppl 1:189-93.

26. Milanov I., Georgiev D., Kmetska K., Jordanova L., Topalov N. Prevalence of multiple sclerosis in Bulgaria. Neuroepidemiology. 1997; 16(6):304-7.

27. Milanov I., Topalov N., Kmetski T. - Prevalence of multiple sclerosis in Gypsies and Bulgarians. Neuroepidemiology. 1999; 18(4):218-22.

28. Milonas I., Tsounis S., Logothetis I. - Epidemiology of multiple sclerosis in northern Greece. Acta Neurol Scand. 1990; 81(1):43-7.

29. Piperidou H.N., Heliopoulos I.N., Maltezos E.S., Milonas I.A. Epidemiological data of multiple sclerosis in the province of Evros, Greece. Eur Neurol. 2003; 49(1):8-12.

30. Zeqiraj K., Kruja J., Kabashi S., Muçaj S. - Epidemiological characteristics and functional disability of multiple sclerosis patients in Kosovo. Med Arh. 2014; 68(3):178-81.

31. Gavrilenko A.A., Evdokimova Z.S., Vasikovskaia G.A., Boľko A.N. [Epidemiology of multiple sclerosis in the Primosky Krai and Far East regions]. Zh Nevrol Psikhiatr Im S S Korsakova. 2012; 112(2 Pt 2):5-8.

32. Pozzilli C., Romano S., Cannoni S. - Epidemiology and current treatment of multiple sclerosis in Europe today. J Rehabil Res Dev. 2002; 39(2):175-85.

33. Sundström P., Nyström L., Forsgren L. - Incidence (1988-97) and prevalence (1997) of multiple sclerosis in Västerbotten County in northern Sweden. J Neurol Neurosurg Psychiatry. 2003; 74(1):29-32.

34. Salzer J., Svenningsson A., Sundström P. - Season of birth and multiple sclerosis in Sweden. Acta Neurol Scand. 2010; 122(1):70-3.

35. Kurtzke J.F. - Further features of the Fennoscandian focus of multiple sclerosis. Acta Neurol Scand. 1974; 50(4):478-502.

36. 14th Italian Congress of Neuroepidemiology. Milan, Italy, November 21-22, 2014: Abstracts.

37. Verdeş F., Petrescu A., Cernescu C. - Epidemiologic survey of multiple sclerosis in the Bucharest city and suburban area. Acta Neurol Scand. 1978; 58(2):109-20.

38. Morariu M., Alter M., Harshe M. - Multiple sclerosis in Transylvania: a zone of transition in frequency. Neurology. 1974; 24(7):673-9.

39. Bălaşa R.F.C., Dan M., Motataianu A., Balaianu M., Chebut C., Constantin V., Bajko Z., Pascu I. - The prevalence of multiple sclerosis in Mures County, central Romania. Revista Romana de Neurologie 2007. p. 80-4. 
40. Kurtzke J.F. - Geographic distribution of multiple sclerosis: An update with special reference to Europe and the Mediterranean region. Acta Neurol Scand. 1980; 62(2):65-80.

41. Zivadinov R., Iona L., Monti-Bragadin L., Bosco A., Jurjevic A., Taus C., et al. - The use of standardized incidence and prevalence rates in epidemiological studies on multiple sclerosis. A meta-analysis study. Neuroepidemiology. 2003; 22(1):65-74.
42. Flachenecker P., Buckow K., Pugliatti M., Kes V.B., Battaglia M.A., Boyko A., et al. - Multiple sclerosis registries in Europe - results of a systematic survey. Mult Scler. 2014; 20(11):1523-32.

43. Kurtzke J.F. - A reassessment of the distribution of multiple sclerosis. Acta Neurol Scand. 1975; 51(2):137-57. 